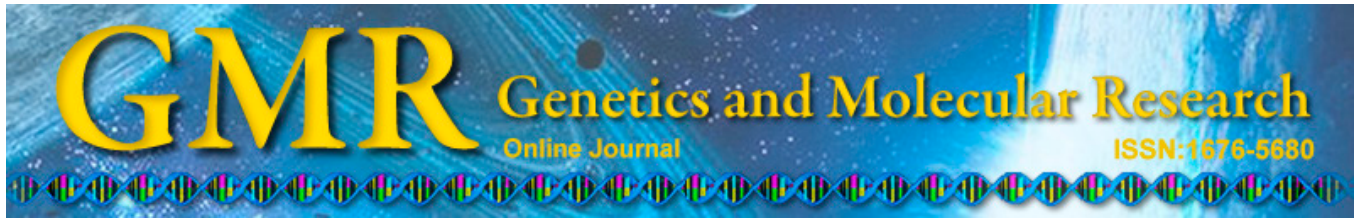

\title{
Cloning and expression of the sucrose transporter gene PSSUT1 from tree peony leaf
}

\author{
Y.H. Li*, T. Guo*, Y. Cui, Y. Li and D. He \\ Department of Ornamental Horticulture, College of Forestry, \\ Henan Agricultural University, Zhengzhou, China \\ *These authors contributed equally to this study. \\ Corresponding author: D. He \\ E-mail: hedan990111@hotmail.com
}

Genet. Mol. Res. 14 (4): 12406-12415 (2015)

Received April 19, 2015

Accepted August 13, 2015

Published October 16, 2015

DOI http://dx.doi.org/10.4238/2015.October.16.7

\begin{abstract}
This study reports the cloning of a sucrose transporter gene, PSSUT1, from the leaf of tree peony (Paeonia suffruticosa Lind. $\mathrm{cv}$ 'Huhong'). Expression patterns were examined in different organs and at different developmental stages. The full-length cDNA of PSSUT1 consisted of a 2001-bp sequence containing a 1557-bp open reading frame, encoding 519 amino acids with a conserved domain typical of the glycoside-pentoside-hexuronide superfamily. The amino acid sequence of PSSUT1 in tree peony shared high homology with that of other plants. At different developmental stages, PSSUT1 was expressed in roots, stems, leaves, and petals. Its expression level in stems was 10.9-fold higher than in petals at the flowering stage. Expression of PSSUT1 at the flowering stage was highest during flower development. The significant differences in PSSUT1 expression observed among developmental stages and organs were closely related to changes in sucrose content during flower opening. These results form the basis for further research on the molecular mechanisms of carbohydrate metabolism and transport during flower development in tree peony.
\end{abstract}

Key words: Tree peony; Sucrose transporter genes; Developmental stage; Cloning and expression 


\section{INTRODUCTION}

Sucrose is the main form of photosynthetic product transported from source organs to library organs. It plays an important role in regulating plant growth process and affecting crop yield and the quality of economic organs (Lalonde et al., 2004; Kühn and Grof, 2010). Sucrose transportation includes two different pathways in higher plants: the symplastic pathway and apoplastic pathway (Ruan and Patrick, 1995; Zhang et al., 2006). The apoplastic pathway requires specific sucrose transporters (SUTs) to facilitate sucrose transport (Sauer, 2007; Rennie and Turgeon, 2009). In recent years, genes that encode sucrose transporters have been identified and characterized in many plants, including Bambusa oldhami, Doritaenopsis hybrid, and Pyrus bretschneideri (Gao et al., 2010; Zhang et al., 2011, 2013a); these studies provide ample information on sucrose apoplastic transport and sucrose transporters. When the AtSUC gene is expressed in the leaves and roots of Manihot esculenta, the level of mRNA fluctuates with the circadian rhythm (Li et al., 2012). AtSUT2 is expressed in the vascular tissues of leaves of Arabidopsis thaliana, and the OSSUT1 promoter in rice is expressed in flag leaves and sprouted grains, suggesting that these genes are involved in the long-distance transport of sucrose (Gottwald et al., 2000).

At present, trends in rates of photosynthesis and dynamic changes in carbon and nitrogen metabolism in roots of tree peony have been discussed (Zhai et al., 2008; Liu et al., 2008), and the sucrose content and distribution in different organs during flower opening have been analyzed (Shi et al., 2009; Zhang et al., 2013b). Sugar transporters may constitute an efficient machinery for promoting sugar uptake or storage in important sinks. To date, however, the cloning and expression of a sucrose transporter gene in tree peony have not been reported. To gain better a understanding into the regulation of sucrose assimilation from source tissues to developing organs and the mechanism of flower development in tree peony, it is necessary to clone and characterize SUT genes. In this study, the full length of sucrose transporter gene was cloned from the leaf of the Paeonia suffruticosa cultivar 'Huhong', the expression of the PSSUT1 in various organs at different developmental stages was determined, and the predicted structure of the transporter was analyzed by comparison to known sucrose transporters from other species.

\section{MATERIAL AND METHODS}

\section{Plant materials}

'Huhong', a cultivar of P. suffruticosa (grafted with the root of P. suffruticosa cultivar 'Fengdan'), was used as the experimental material in this study. The plants were cultivated in a research station of Henan Agricultural University. Healthy and uniform peony plants were chosen and sampled in the peach period (April 1, 2013), the flowering period (April 18, 2013), the withering period (April 28, 2013), and the bulbil differentiation period (June 21, 2013). Root (principal root), phloem in stem (middle section), healthy functional leaves, and 3-5 layers of petals (from edges, inward) were harvested for further experiments. All samples were quickly cleaned and frozen in liquid nitrogen after harvesting and stored at $-70^{\circ} \mathrm{C}$ until further use. 


\section{Total RNA extraction and cDNA synthesis}

Fresh leaves $(0.5 \mathrm{~g})$ were collected and ground into powder in liquid nitrogen. Total RNA was extracted using an improved CTAB method (Meng et al., 2006). The RNA was then treated with DNase I according to the manufacturer protocol (Takara, Japan) so as to remove contamination by genomic DNA. An aliquot of RNA was quantified by light absorbance at $260 \mathrm{~nm}$ and then electrophoretically separated on a $1.0 \%$ agarose gel to verify integrity. The first-strand cDNA was synthesized from $2.0 \mu \mathrm{g}$ total RNA using a RevertAid First-Strand cDNA Synthesis Kit (Fermentas, Ontario, Canada), according to the manufacturer protocol.

\section{Molecular cloning of PSSUT1 cDNA}

Two highly conserved regions were identified by amino acid sequence alignment of existing cloned sucrose transporters from plants. The cDNA samples derived from leaves of tree peony cv. 'Huhong' were amplified using primers deduced from conserved regions. The resulting PCR fragment was cloned into Pgem-T Easy vector (Promega, Madison, WI, USA) and sequenced. The remainder of the cDNA was produced using 5'- and 3'-RACE PCR, performed using nested PCR with the gene-specific primers derived from the newly isolated partial fragment and the SMARTer RACE cDNA amplification kit (Clontech, Palo Alto, CA, USA). Amplification products of 5'-RACE and 3'-RACE were cloned into pGEM-T Easy vector (Promega) and sequenced. Following end-to-end PCR, the resulting DNA fragment was sequenced and then BLAST searched against the GenBank database.

\section{Sequence analysis}

Nucleotide sequence alignment was analyzed using the DNAMAN 6.0 software (Lynnon Biosoft, USA). The amino acid sequence was predicted through BLASTp on the NCBI website (http://www.ncbi.nlm.nih.gov/). Physicochemical characteristics of the protein were recorded using the ProtParam online software (http://web.expasy.org/protparam/). Transmembrane regions were predicted using TMpred server (http://www.ch.embnet.org/software/TMPRED_form.html). SignalP tools (http://www.cbs.dtu.dk/services/SignalP/) were used to predict the signal peptide. PSORT (http://psort.hgc.jp/form.html) was used to predict subcellular localization. SOPMA (http://npsa-pbil.ibcp.fr/cgi-bin/npsa_automat.pl?page=npsa_sopma.html) was used to analyze secondary structure. Phylogenetic analyses were conducted using the MEGA 5.0 software with the neighbor-joining method followed by phylogeny test options of 1000 bootstrap replicates.

\section{Real-time qPCR assay}

Total RNA was extracted from various organs of tree peony at different developmental stages and reverse-transcribed to cDNA. The resulting cDNA was amplified with RT-qPCR primers P6 and P7 (Table 1), which were designed according to the PSSUT1 cDNA intermediate fragment sequences. Resulting amplified fragments were $105 \mathrm{bp}$ in length. Using the peony Actin gene as a reference (Wang et al., 2012), and Act-F and Act-R as primers (Table 1), 134-bp fragments were amplified. qRT-PCR analysis was performed on a BIO-RAD iQ2 Real-Time Detection System with Power x SYBR Real-time PCR Premixture Kit (BioTeke Corporation, Beijing), according to manufacturer protocol. The reaction mixture contained $10 \mu \mathrm{L} 2 \mathrm{X}$ Premix, 
$0.4 \mu \mathrm{L}$ of each primer $(10 \mu \mathrm{M}), 2 \mu \mathrm{L}$ cDNA, and $7.2 \mu \mathrm{L} \mathrm{ddH_{2 }} \mathrm{O}$, for a total reaction volume of $20 \mu \mathrm{L}$. The reaction conditions were as follows: initial denaturation and DNA polymerase activation at $95^{\circ} \mathrm{C}$ for $2 \mathrm{~min} ; 40$ cycles of denaturation at $95^{\circ} \mathrm{C}$ for $15 \mathrm{~s}$, annealing at $60^{\circ} \mathrm{C}$ for $20 \mathrm{~s}$, and extension at $72^{\circ} \mathrm{C}$ for $20 \mathrm{~s}$. For each sample, three replicates were performed. The relative transcript levels were calculated by the $2^{-\Delta \Delta C t}$ method (Livak and Schmittigen, 2001).

Table 1. Primers used in gene cloning and expression analysis.

\begin{tabular}{ll}
\hline Primer name & Primer sequence \\
\hline P1 & 5'-GCC(T)GGT(A)GTTCAA(G)TTC(T)GG-3' \\
P2 & 5'-A(G)CCCATCCAA(G)TCA(T)GT A(G)TC-3' \\
P3 & 5'-GGAGGAAGGGAAATCAGC-3' \\
P4 & 5'-CGCCGAATAGGTGACCGAGAT-3' \\
P5 & 5'-AAATCGGGAAGAGCATCGGTCA-3' \\
P6 & 5'-CGGTAACAACCAGAAACG-3' \\
P7 & 5'-TACATCTGCCTGAACGAA-3' \\
B26 & 5'-GACTCTAGACGACATCGA(T) ${ }_{17}$-3' $^{\prime}$ \\
Oligo(dT) & 5'-TTTTTTTTTTTTTTTT-3' \\
UPM & 5'-CTAATACGACTCACTATAGGGCAAGCAGTGGTATCAACGCAGAGT-3' \\
Act-F & 5'-GGTCTATTCTTGCTTCCCTCAG-3' \\
Act-R & 5'-GAACTCACTATCAAAC CCTCCAG-3' \\
\hline
\end{tabular}

\section{RESULTS}

\section{Cloning the PSSUT1 gene}

Reverse transcription of total RNA followed by amplification yielded a fragment of the expected size, around $800 \mathrm{bp}$ (Figure 1). The band was subcloned and sequenced, revealing a 825-bp fragment. Primers for 3'-RACE and 5'-RACE were designed according to the intermediate fragment sequences, and nested PCR was carried out with those primers. Electrophoresis of the target segment in agarose gel revealed amplification bands of $950 \mathrm{bp}$ for 3'-RACE and approximately $450 \mathrm{bp}$ for 5'-RACE. Sequenced results revealed the length of the 3'-RACE fragment to be $981 \mathrm{bp}$ and the 5'-RACE fragment to be $422 \mathrm{bp}$ (Figure 1).

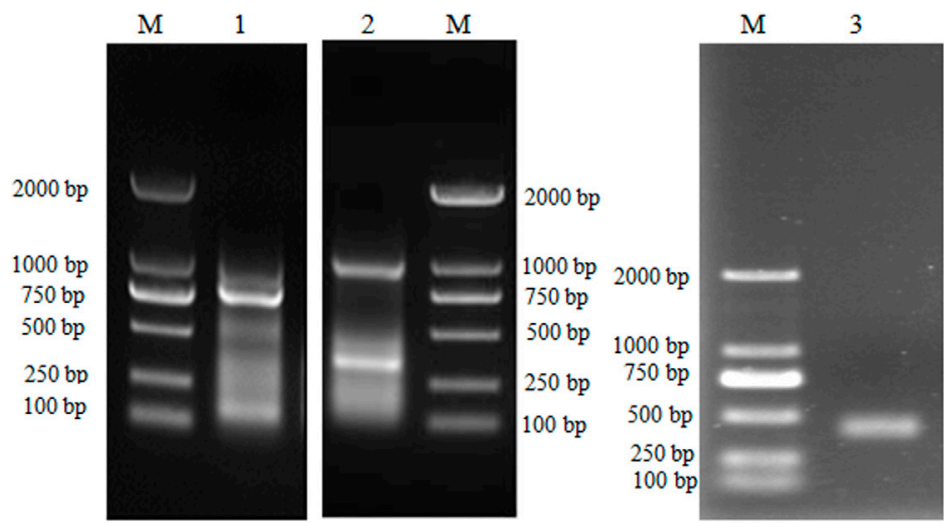

Figure 1. Agrose gel electrophoresis of the PSSUT1 amplification product of tree peony. Lane $M=$ DNA marker DL2000; lane 1 = middle fragment; lane 2 = 3'-RACE; lane 3 = 5'-RACE. 
The full-cDNA sequence was obtained by combining the information contained in the 5'- and 3'-fragments; it was named PSSUT1 (GenBank accession No. KC542395). Sequence analysis revealed a 1557-bp open reading frame (ORF) encoding a 519-amino acid protein. The PSSUT1 gene shares more than $70 \%$ identity with SUT genes from other species.

\section{Peony PSSUT1 homology and phylogenetic analysis}

BLAST indicated that the deduced amino acid sequence of PSSUT1 shared roughly $65,64,63,68,66,65,67,68$, and $69 \%$ identity, respectively, with homologous sequences from Medicago truncatula (AFM28284), A. thaliana (NP 173685), Brassica oleracea (AAL58072), Hevea brasiliensis (CAM34330), Glycine max (NP_001236298), Pisum sativum (AAD41024), Juglans regia (AAU11810), Populus trichocarpa (ADW94613), and Vitis vinifera (AAF08331). Homology with SUC27 of $V$. vinifera was 69\% (Figure 2), the highest among these species, indicating that this gene is relatively conserved.

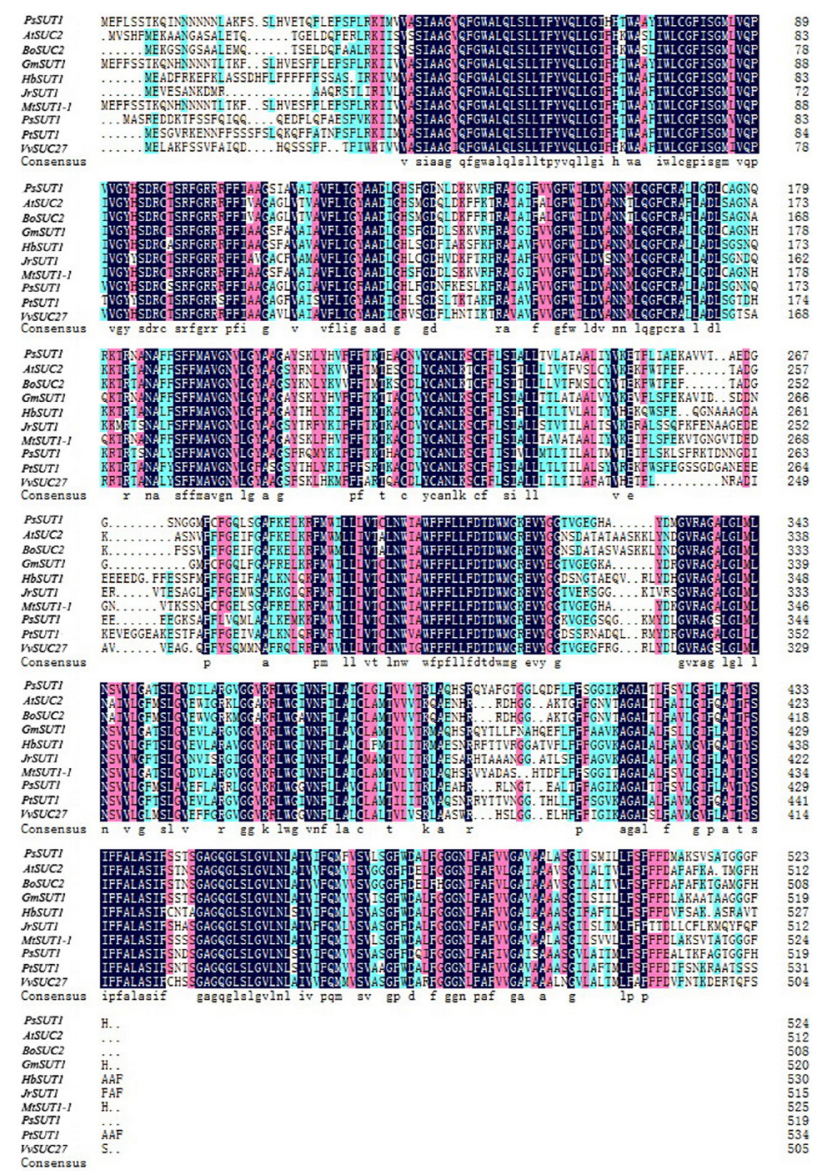

Figure 2. Alignment of amino acid sequence of PSSUT1 from tree peony and other plants. Conserved residues in this alignment are shaded in dark blue (homology level $=100 \%$ ), pink (homology level $\geq 75 \%$ ), light blue (homology level $\geq 50 \%$ ). 
Using amino acid sequence homology to analyze the evolutionary relationships between PSSUT1 and the SUT subfamilies, a neighbor-joining phylogenetic tree was constructed using MEGA4.0 (Tamura et al., 2007). The resulting phylogenetic tree was divided into five subfamilies: SUT1, SUT2, SUT3, SUT4, and SUT5 (Figure 3). SUT1 included J. regia, V. vinifera, Solanum tuberosum, Nicotiana tabacum, and Beta vulgaris. P. suffruticosa and J. regia were located in the same branch, revealing a close evolutionary relationship between them, with $V$. vinifera being the second most closely related species.

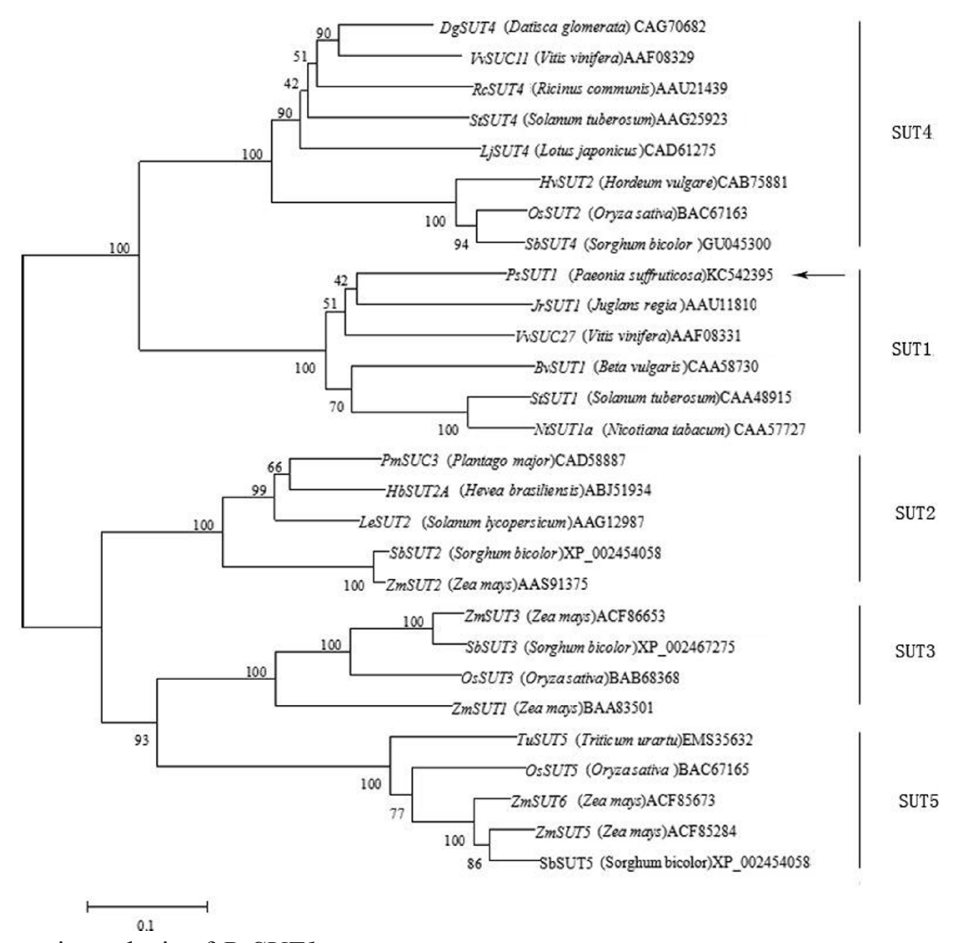

Figure 3. Phylogenetic analysis of PSSUT1.

\section{Characterization of the protein encoded by PSSUT1}

PSSUT1 encodes a proteins with the predicted molecular formula $\mathrm{C}_{2546} \mathrm{H}_{4016} \mathrm{~N}_{650} \mathrm{O}_{686} \mathrm{~S}_{25}$, which is composed of 22 types of amino acids and has a molecular mass of $55.5 \mathrm{kDa}$ and isoelectric point of 9.02. Analysis of protein hydrophobicity showed that the maximum coefficient was 3.311 (located in the 229th amino acid residue), the minimum was -2.844 (located in the 176th amino acid residue), and most regions were hydrophobic. Subcellular localization predicts a possibility of the protein being localized in the cytoplasmic membrane, mitochondria, Golgi apparatus, and endoplasmic reticulum of $0.800,0.654,0.400$ and 0.300 , respectively. Therefore, the location of the PsSUT1 protein was inferred as the cytoplasmic membrane. Analysis revealed no obvious signal peptide. The secondary structure of the PsSUT1 protein consists of $\alpha$-helix, extended strand, random coil and $\beta$-turn, with percentages of 42.20, 18.30, 36.42 , and $3.08 \%$. The sucrose transporter is a transmembrane protein; the high content of hydrophobic amino acids is beneficial for the formation of transmembrane domains. 
The predicted membrane topology (by TMPred) of the PsSUT1 protein consisted of 12 transmembrane domains (Figure 4), suggesting that PsSUT1 might be a transmembrane protein. According to NCBI CDS (conserved domain search) analysis, the protein has a typical sugar transporter protein domain belonging to the glycoside-pentoside-hexuronide/sucrose transport subfamily of cation symporter.

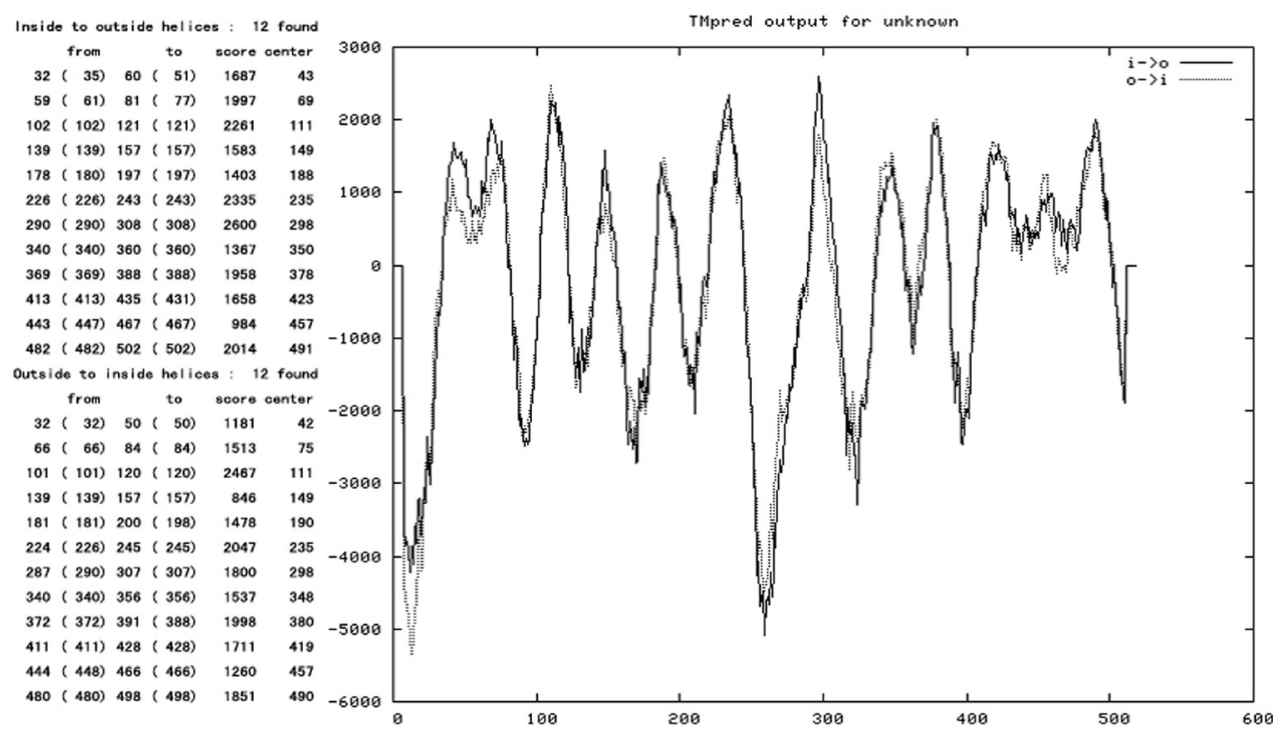

Figure 4. Transmembrane domain analysis of the PSSUT1 protein.

\section{Expression pattern of PSSUT1}

The expression pattern of PSSUT1 in different organs at different developmental stages was analyzed using RT-qPCR with Actin as the reference gene (Figure 5).

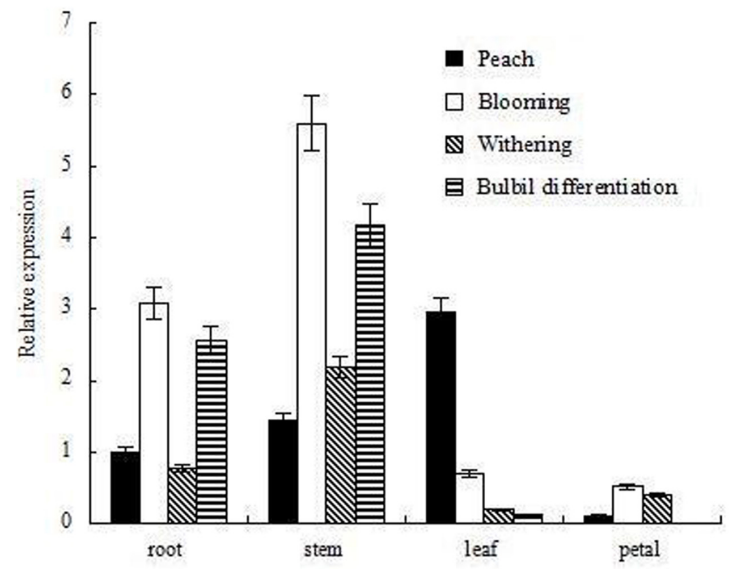

Figure 5. Relative expression level of PSSUT1 in different organs at different development stages. 
There were significant differences in expression of $P_{S S U T 1}$ in different organs at the same stage. The expression level of PSSUT1 in leaves was the highest at the peach stage, and was 27.5 times of petals. At flowering, withering and bulbil differentiation stages, expression level of the PSSUT1 gene was the highest in stems, while the lowest in petals. PSSUT1 expression in roots and stems had the same variation tendency; both of them were the highest at flowering periods. While PSSUT1 expression showed a gradual decline in leaves, expression level of PSSUT1 decreased by $95.9 \%$ from bulbil differentiation stage to peach stage. In petals, PSSUT1 expression was highest at the flowering stage, which was $379 \%$ higher than that at the peach stage. With petal senescence, expression of the PSSUT1 gene declined at the withering stage.

\section{DISCUSSION}

Since the first plant SUT gene was cloned from spinach in 1992, many genes have been cloned from plants. These genes encoding sucrose transporter proteins belong to a multigene family, as indicated by significant similarity at the amino acid level (Williams et al., 2000). According to phylogenetic analyses, plant SUTs have been classified into five subfamilies. The SUT1 subtribe is expressed in dicots, the SUT2 and SUT4 subtribes are expressed in both dicots and monocots, and the SUT3 and SUT5 subtribes, which were cloned from maize (Aoki et al., 1999) and sorghum (Kühn and Grof, 2010), are particular to monocots. All sucrose transporters of the SUT1 subtribe, which derived from dicots, are typical membrane protein with higher affinity and lower transporting capacity including 12 transmembrane regions, the $\mathrm{N}$ - and $\mathrm{C}$-termini are localized to the cytoplasmic side.

In this experiment, we cloned the full-length PSSUT1 cDNA from tree peony leaves. PSSUT1 cDNA contained a 1557-bp open reading frame encoding a 519-amino acid protein with a relative molecular mass of $55.5 \mathrm{kDa}$ and an isoelectric point of 9.02. The PsSUT1 protein contained 12 transmembrane domains, a distinct characteristic of the GPH/cation symporter family (Figure 4). A neighbor-joining phylogenetic tree of PSSUT1 and homologous SUT genes was constructed, resulting in a phylogenetic tree divided into five distinct subtribes (Figure 3). In the SUT1 subtribe PSSUT1 shared the closest genetic distance to J. regia, and second-closest to $V$. vinifera, while the genetic distances between PSSUT1 and most members of the SUT3 and SUT5 subtribes were greater. The amino acid sequence of PsSUT1 showed the greatest degree of homology to those of the SUT1 subtribe, while it had low homology to other subtribes. The protein encoded by PSSUT1 has 12 typical transmembrane regions, consistent with the characteristics of SUT1 subtribe proteins, which are membrane binding proteins. PSSUT1 showed high degrees of homology to homologs in M. truncatula, H. brasiliensis, P. trichocarpa, and $V$. vinifera. Furthermore, phylogenetic analysis also showed that the PsSUT1 protein had the closest genetic relationship with the sucrose transporter from J. regia, which belongs to SUT1 subtribe, thereby classifying PSSUT1 as a member of the SUT1 subtribe.

Long distance transport of sucrose in phloem is beneficial for the growth and development of plants. Sucrose transporter proteins play a key role in the phloem loading and unloading of sucrose (Sun et al., 2010; Reinders et al., 2012). In higher plants, due to different expression patterns, SUT subtribes exibit significant differences in function. BoSUT2 of Dendrocalamopsis oldhami is expressed in leaf, leaf sheath, and root, while its expression is not detected in caulicle. These results reveal that BoSUT2 has obvious tissue-specific char- 
acteristics (Gao et al., 2010). Similarly, PbSUT1 of pear is expressed mainly in fruit (Yao et al., 2010; Zhang et al., 2013a). Our expression analysis of PSSUT1 at four growth stages revealed expression in roots, stems, leaves, and petals, but there were no obvious tissue-specific characteristics. PSSUT1 expression level was higher in tissues that accumulate more carbohydrates, such as stems and roots, while it was low in petals, depending on developmental stage. It seems that tree peony blossoms need more energy, so that sucrose stored in roots is transported long distances through the phloem in order to provide nutrients for plants growth and flowering. PSSUT1 expression level showed a trend of gradual decline in leaves. At the peach stage, expression was highest in leaves and lowest in petals. Leaves are important sink tissues before blooming, so they need increased energy to satisfy the requirements of growth and photosynthesis and provide sufficient energy for blooming. In petals, PSSUT1 expression was highest at the flowering stage, because petals consume more nutrients and energy when blooming. There were significant differences in PSSUT1 expression during the tree peony blossom stage. PSSUT1 is expressed not only in sink tissues, but also in source tissues. This reveals the dual function of PSSUT1, which is able to mediate both sucrose unloading and phloem loading. Indeed, many studies have found that $S U T 1$ is expressed in both sink tissues and source tissues, and these carrier proteins are thought to play dual functions: regulating the loading in source tissues and unloading in sink tissues of photoassimilates (mainly sucrose) in apoplastic phloem transmembrane pathway (Lalonde et al., 1999; Kühn et al., 2003). For example, StSUT1 of Solanum tuberosum, which is responsible for assimilation loading, also plays an important role in tuber phloem unloading (Kühn et al., 2003). SUT genes cloned from Galega officinalis (Li et al., 2011), Malus pumila (Peng et al., 2011), Triticum aestivum (Deol et al., 2013), and Leymus chinensis (Su et al., 2013) are expressed both in source leaves and sink tissues such as seeds, fruits, and stems. The present experiment revealed that PSSUT1 was critical to the allocation and utilization of sucrose in tree peony. Sucrose transporters are responsible for the absorption and transportation of carbohydrate, and so are necessary to the long distance transport of sucrose (Riesmeier et al., 1994). At flowering, withering, and bulbil differentiation stages in tree peony, PSSUT1 expression was the highest in stems; this demonstrated that PSSUT1 was involved in the phloem transport of sucrose. Therefore, the functions of PsSUT1 may be more complicated than the transport of sucrose; there remains more information to be discovered experimentally.

\section{ACKNOWLEDGMENTS}

Research supported by the Special Science and Technology Foundation of Henan Province (Grant \#102102110033) and the Zhengzhou Key Project of Science and Technology (\#131PZDGG325).

\section{REFERENCES}

Aoki N, Hirose T, Takahashi S, Ono K, et al. (1999). Molecular cloning and expression analysis of a gene for a sucrose transporter in maize (Zea mays L.). Plant Cell Physiol. 40: 1072-1078.

Deol KK, Mukherjee S, Gao F, Brûlé-Babel A, et al. (2013). Identification and characterization of the three homeologues of a new sucrose transporter in hexaploid wheat (Triticum aestivum L.). BMC Plant Biol. 181: 1-15.

Gao ZM, Yang XW, Peng ZH, Li XP, et al. (2010). Molecular characterization and subcellular localization of BoSUT2 from Bambusa oldhamii. Sci. Silv. Sin. 46: 45-50. 
Gottwald JR, Krysan PJ, Young JC, Evert RF, et al. (2000). Genetic evidence for the in planta role of phloem-specific plasma membrane sucrose transporters. PNAS 97: 13979-13984.

Kühn C and Grof CPL (2010). Sucrose transporters of higher plants. Curr. Opin. Plant Biol. 13: 288-298.

Kühn C, Hajirezaei MR, Fernie AR, Roessner-Tunali U, et al. (2003). The sucrose transporter StSUT1 localizes to sieve elements in potato tuber phloem and influences tuber physiology and development. Plant Physiol. 131: 102-131.

Lalonde S, Boles E, Hellmann H, Barker L, et al. (1999). The dual function of sugar carriers: transport and sugar sensing. Plant Cell 11: 707-726.

Lalonde S, Wipf D and Frommer WB (2004). Transport mechanisms for organic forms of carbon and nitrogen between source and sink. Ann. Rev. Plant Biol. 55: 341-372.

Li J, Wang XM, Dong J, Wang Z, et al. (2011). Isolation and construction expression vector of GoSUT in Galega orientalis. Chin. J. Grassl. 33: 39-45.

Li ZX, Wang HY, Hu HZ, Lu C, et al. (2012). Expression of sucrose transporters in organs of different developing stages of cassava. Chin. J. Trop. Crops 33: 1204-1207.

Liu ZM, Kong DZ, Li YH, and Yang QS (2008). Dynamic carbon and nitrogen metabolism in roots of potted and field Paeonia suffruticosa 'Luoyanghong'. Sci. Silv. Sin. 9: 162-164.

Livak KJ and Schmittigen TD (2001). Analysis of relative gene expression data using real-time quantitative PCR and $2^{-\Delta \Delta C t}$ method. Methods 25: 402-408.

Meng L, Zhou L, Zhang MS and Dai SL (2006). An efficient and economic method for preparation total RNA of petals. Biotechnology 16: 38-40.

Peng CC, Xu YH, Xi RC and Zhao XL (2011). Expression subcellular localization and phytohormone stimulation of a functional sucrose transporter (MdSUT1) in apple fruit. Sci. Hortic. 128: 206-212.

Reinders A, Sivitz AB and Ward JM (2012). Evolution of plant sucrose uptake transporters. Front. Plant Sci. 3: 1-12.

Rennie EA and Turgeon R (2009). A comprehensive picture of phloem loading strategies. PNAS 106: 14162-14167.

Riesmeier JW, Willmitzer L and Frommer WB (1994). Evidence for an essential role of the sucrose transporter in phloem loading and assimilate partitioning. EMBOJ 13: 1-7.

Ruan YL and Patrick JW (1995). The cellular pathway of post phloem sugar transport in developing tomato fruit. Planta 196: 434-444.

Sauer N (2007). Molecular physiology of higher plant sucrose transporters. FEBS Letters 581: 2309-2317.

Shi GA, Guo XF, Zhang GH and Bao MZ (2009). Analysis of sugar metabolism during florescence and flower senescence of tree peony petal. Acta Hortic. Sin. 36: 1184-1190.

Su M, Li XX, Li XF, Cheng LQ, et al. (2013). Molecular characterization and defoliation-induced expression of a sucrose transporter lcsut1 gene in sheep grass (Leymus chinensis). Plant Mol. Biol. Rep. 31: 1184-1191.

Sun Y, Reinders A, LaFleur KR, Mori T, et al. (2010). Transport activity of rice sucrose transporters OsSUT1 and OsSUT5. Plant Cell Physiol. 51: 114-122.

Tamura K, Dudley J, Nei M, and Kumar S (2007). MEGA4: Molecular Evolutionary Genetics Analysis (MEGA) software version 4.0. Mol. Biol. Evol. 24: 1596-1599.

Wang YJ, Dong L, Zhang C and Wang XQ (2012). Reference gene selection for real-time quantitative PCR normalization in tree peony (Paeonia suffruticosa Andr). J. Agr. Biotechnol. 20: 521-528.

Williams LE, Lemoine R and Sauer N (2000). Sugar transporters in higher plants: a diversity of roles and complex regulation. Trends Plant Sci. 5: 283-290.

Yao GF, Zhang SL, Cao YF, Liu J, et al. (2010). Characteristics of components and contents of soluble sugars in pear fruits from different species. Sci. Agr. Sin. 43: 4229-4237.

Zhai M, Li YH and Yang QS (2008). Comparison of photosynthetic characteristics between potted and field Paeonia suffruticosa Andr. Acta Hortic. Sin. 35: 251-256.

Zhang C, Zhou LP, Luo XY, Sun XM, et al. (2011). Relative cold-induced flowering arouse fluctuation on carbohydrates and expression of genes related to sugar transport in Doritaenopsis hybrid. Sci. Agr. Sin. 44: 1670-1677.

Zhang H, Zhang S, Qin G, Wang L, et al. (2013a). Molecular cloning and expression analysis of a gene for sucrose transporter from pear (Pyrus bretschneideri Rehd.) fruit. Plant Physiol. Biochem. 73: 63-69.

Zhang KM, Wang CL, Wang D, Li Y, et al. (2013b). Dynamic change of sugar component in stems and roots of tree peony at different developmental stages. J. Henan Agr. Univ. 47: 284-288.

Zhang XY, Wang XL, Wang XF, Xia GH, et al. (2006). A shift of phloem unloading from symplasmic to apoplasmic pathway is involved in developmental onset of ripening in grape berry. Plant Physiol. 142: 220-232. 